\title{
REPLACEMENT OF THE YELLOW DIXIE CUP
}

\author{
Capt Eileen Ancman \\ Lt Chris Poprik \\ HSC/XRS \\ Brooks AFB, TX \\ (210)536-4456
}

\begin{abstract}
The upgrade and improvement of life support systems has always been a priority for the United States Air Force. A study is currently underway to determine the feasibility of replacing the emergency passenger protective breathing device used on USAF transport aircraft. The present system, the "yellow dixie cup", provides supplemental oxygen in the event of a rapid decompression. The system is not designed to provide protection in the event of a fire or toxic fumes resulting from a cargo spill. The proposed replacement, utilizing either a positive pressure closed loop or filter based open loop system, will provide protection during any of these circumstances. In addition to personnel safety considerations, the Air Force continually strives to improve product reliability and maintainability. Approximately one third of the yellow dixie cups must be replaced annually, whether used or not. Specifications for the new mask include a requirement for an active five year shelf life. The effort, known as the Passenger Smoke and Fume Protective Device (PSFPD) study, is presently in the market survey phase, where the potential for buying an off-the-shelf item is under evaluation. This type of procurement, a Nondevelopmental Item (NDI) acquisition, is a highly cost effective method of meeting DoD needs. The market survey includes conducting evaluations to develop a performance-based specification to be used for the procurement if a suitable replacement is found. The projected acquisition is planned for FY1995. The commercial aviation industry, which also uses the yellow dixie cup, has taken a great interest in the results of the study.
\end{abstract}

\section{INTRODUCTION}

In an effort to improve both passenger safety and product reliability and maintainability (R\&M), the Air Force has initiated a study of emergency passenger protective breathing devices. This effort, known as Passenger Smoke and Fume Protective Device (PSFPD) study, will compare and evaluate more recent technology with respect to the existing system, the "yellow dixie cup." The yellow dixie cup, identical in form, fit, and function to the commercial aviation counterpart, serves as a provider of emergency oxygen during cabin depressurization. The system is not designed to provide protection in the event of a fire or toxic chemical spill. In each case, reactive operational procedures (ie., descent, extinguish fire, circulation of air, etc.) must be relied on to minimize passenger exposure levels. The PSFPD study is the first in a series of Air Force actions to upgrade passenger safety on transport aircraft. A new system, with a higher level of protection, would complement proactive safety measures (ie., quality handling and packaging of hazardous materials), as well as provide reactive response upon donning.

In keeping with the Integrated Weapons System Management (IWSM) philosophy of life cycle cost reduction, the study includes a cost benefit analysis of both acquisition and R\&M costs. The cost of maintaining the current system is high in comparison to the actual cost of equipment. By replacing the system, the Air Force will improve R\&M. The new system is required to have a five year (minimum) shelf life, an improvement over the yellow dixie cup's three year life span.

With respect to an environment with fewer resources, yet growing needs, the acquisition process must be responsive to alternative methods for cost reduction. Non-developmental Item (NDI) acquisition is an existing and proven vehicle for procurement cost reduction with respect to existing technology. This study will provide the data to determine if a product can be purchased off-the-shelf through the evaluation of commercially available products and an assessment of requirements. 


\section{BACKGROUND}

Air Mobility Command (AMC) is responsible for the operation and maintenance of the aircraft in which emergency passenger oxygen equipment is utilized. AMC's primary mission involves the transport of troops and materials. In keeping with the historical need for employment flexibility, the aircraft are designed to conduct both missions concurrently, thus giving rise to the need for a mask that provides protection from hazards such as smoke and toxic fumes resulting from inflight mishaps. In line with the concept of improved systems at lower costs, any proposed replacement would be required to meet or exceed the current capability of the yellow dixie cup, including lower life cycle cost, ease of use, and stowage size. The storage requirement is critical due to the configuration of the oxygen supply on-board the aircraft. If the new mask can not be packaged to fit into the existing configuration of the aircraft, modifications would be necessary and prohibitively expensive. This is not an option for consideration.

In the current configuration, the yellow dixie cup is employed as either a drop-down system connected to the aircraft supply of oxygen (identical on both military and civilian aircraft) or as an independent unit, attached to a stand alone bottle of oxygen. The portable unit, known as a Passenger Oxygen Kit (POK), is distributed to each individual boarding the aircraft. To reduce logistical and maintenance costs, a single replacement (as opposed to two separate systems) should be capable of interfacing with either configuration.

Using these requirements as a corner stone, the Air Force is conducting a comparative analysis of the commercially available passenger masks to determine whether or not existing technologies can satisfy the expanded need of AMC. The market survey is the first step in evaluating the potential of an NDI procurement. This portion of the study includes limited evaluations of potential products designed to highlight the current requirements and assist in the finalization of the replacement system specification. The survey has no intent to eliminate any products from later competition; its purpose is to determine if off-the-shelf hardware can meet AMC's most critical requirements. As not all of the requirements are deemed critical, the survey also serves the purpose of evaluating whether or not a "noncritical " requirement should be considered a "necessity" or a "goal". Modifications to requirements that do not compromise the ability of the final product to deliver the required protection enable the use of an NDI vehicle for procurement, thereby providing a savings to the government.

\section{PROGRAM DESCRIPTION}

The PSFPD study is being conducted by the Studies and Analysis Division of the Human Systems Center (HSC/XRS) at Brooks AFB TX. HSC/XRS actively sought support for the effort after AMC presented the need for a replacement item. The Productivity, Reliability, Availability, Maintainability office (PRAM) provided the funding for the evaluation, and PE65808 funds were utilized to put Battelle on contract to assist with the effort. The study includes researching the current technology through a literature search followed by the market survey evaluation. The data will then be used to complete a draft specification, decision matrix, and cost benefit analysis for AMC, where the decision will be made on a procurement effort.

The first step in the process of evaluation involved contacting the public in as wide a dissemination as possible. An announcement was placed in the Commerce Business Daily (CBD) stipulating key requirements, including cost and basic function. Fourteen inquiries were received, and each vendor was subsequently invited to attend a meeting to present their products.

Eleven vendor's participated in the meeting, presenting items ranging from off-the-shelf hardware to developmental prototypes. Although a variety of systems were presented, each fell into one of two categories: 1) closed loop systems providing supplemental oxygen or 2) open loop, filter based systems.

After seeing the presentations, Air Force personnel concluded that the market held the potential for a replacement system. Each vendor that presented was then invited to participate in a follow-on evaluation involving laboratory testing. Of the eleven participants, seven submitted samples for evaluation.

One of the most critical tasks in the study is the refinement/ delermination of quantitative requirements. AMC defined some of the more general needs; however, issues such as the fire and toxic environment (ie., concentration levels, breakthrough, etc.) had yet to be analyzed. While researching on-board fire smoke 
and fume levels, an existing specification on the subject was found. The European Organisation for Civil Aviation Equipment (EUROCAE) published the Minimum Operational Performance Specification for Passenger Protective Breathing Equipment in April 1991. The Air Force studied the document, and found the contents to be conducive to application for DoD needs. Although the elements of the specification were based on a study of commercial aircraft, the aircraft structure and material framework is the same, or similar, throughout industry and the DoD. This led to the consequential acceptance of the studied levels of exposure to smoke and fume by-products, as well as the protection factors necessary to insure safety.

The toxic environment presented yet another challenge. Ideally, the Air Force needs to protect passengers from the $1000+$ compounds listed in AFR 71-4, a compilation of hazardous materials transported by the DoD. Many of these items rarely, if ever, are actually carried on-board aircraft. This led to the need to narrow the list to a more realistic representation of AMC cargo. Air Force engineers, as well as contracting specialists, researched the requirement, and with the aid of the Defense Logistics Agency (DLA) were able to compile a list of the most representative compounds carried. For each compound, human exposure data was then researched and subsequent base level exposure and protection levels were set. The actual calculation of subject environment versus protection factors has yet to be determined. This requirement will be developed by one of the testing agencies, and later evaluated by Air Force personnel.

With all other requirements defined, an Evaluation Master Plan (EMP) was drafted to document all testing procedures and scheduling. The EMP was organized into two sequential test phases. Phase I, composed of three tests, evaluates the market capability against those requirements that will not change. Phase II evaluates the criteria that must be refined using the market capability as a source of guidance.

In September 1993, vendors submitted products and Phase I of the commercial evaluation began. The first test examined the ability of the masks to withstand environmental fluctuations on-board aircraft. This included a hot/cold sink under various levels of humidity for a period of 120 hours. Test II involved the experimental determination of the protection factor provided by each mask. Test III, the final evaluation of
Phase I, applied only to filter based systems. This test exposed each filter to a variety of by-products from aircraft fires, including carbon monoxide, and measured the amount of time before breakthrough.

Phase II is made up of two separate tests. The first evaluation is composed of human factors integration, work load, and rapid decompression testing. Those units that utilize filters will go through a second set of filter evaluations to aid in the refinement of exposure levels to the various chemicals representative of a hazardous cargo spill.

Once all of the evaluations are complete, the results will be used to complete a draft performance based specification, decision matrix, and cost benefit analysis. This data will be presented to AMC, where the decision will then be made to either: 1) begin a competitive acquisition, 2) turn the effort into a research and development (F\&D) effort, or 3) discontinue the program.

\section{SUMMARY}

In the face of force and budget reductions, the Air Force continues to strive for improvement. To date, the yellow dixie cup has served the purpose of providing passenger protection in the event of rapid decompression. The time has come, however, when need exceeds capability. The Passenger Smoke and Fume Protective Device effort is both a study of market technology and a needs assessment. Once all evaluations are complete, the Air Force will have a specification defining a new product, one that will become a "remove and replace" item for the yellow dixie cup.

With the data collected in the market survey, the Air Force should also know, with a fair amount of certainty, whether or not the market can meet increasing needs. If the technology does exist to meet these needs without modification to aircraft, the procurement effort becomes a simple process. However, if this technology does not exist, the Air Force is a step further in the acquisition process having defined their specific needs in a requirements document, including testing procedures for qualification. 